\title{
CORRELATION-BASED DEBLURRING LEVERAGING MULTISPECTRAL CHROMATIC ABERRATION IN COLOR AND NEAR-INFRARED JOINT ACQUISITION
}

\author{
Majed El Helou, Zahra Sadeghipoor, Sabine Süsstrunk
}

\author{
School of Computer and Communication Sciences, EPFL, Lausanne, Switzerland
}

\begin{abstract}
Joint acquisition of color and near-infrared (NIR) images is of growing interest due to various applications that make use of the additional spectral information. An obstacle to this acquisition is the wavelength-dependent blurring caused by the chromatic aberration of optical lenses. When one of the spectral channels, for example the green channel, is in focus on the sensor plane, the images of the other channels, especially NIR, are blurred. This paper presents a study of spectral-spatial correlations between color and NIR channels and proposes a method to correct for chromatic aberrations. The algorithm we introduce leverages axial chromatic aberration to deblur the NIR image when the color image is in focus. The proposed technique improves image sharpness by $48.8 \%$ on average compared to state-of-the-art results. Moreover, our method generates an NIR image that has a larger depth-of-field compared to an NIR image originally captured in focus.
\end{abstract}

Index Terms - Joint color-NIR imaging, chromatic aberration, multispectral deblurring, spectral-spatial correlation, depth-of-field.

\section{INTRODUCTION}

Silicon sensors of most digital cameras detect both visible (red, green and blue) and NIR light, though the latter is filtered before reaching the sensor in order not to affect the color image [1]. However, the acquisition of NIR radiation is valuable for several applications. NIR information, combined with color images, improves the estimation of shadow regions in images [2], scene recognition [3], illuminationinvariant face recognition [4], analysis of food products, soil, and crops [5-7], as well as low-light imaging [8] and image dehazing [9]. Recent research [10-13] has shown multiple ways of how to jointly acquire visible and NIR images, to realize the full potential of the camera's silicon sensor.

Simultaneous acquisition of color and NIR images increases the captured wavelength range from the visible range (400-700 nm) to cover wavelengths from 700 to $1100 \mathrm{~nm}$ (NIR radiation) as well. However, different wave bands are focused on different points on the optical axis as the refractive index of a simple lens is wavelength dependent (see Fig. 1).

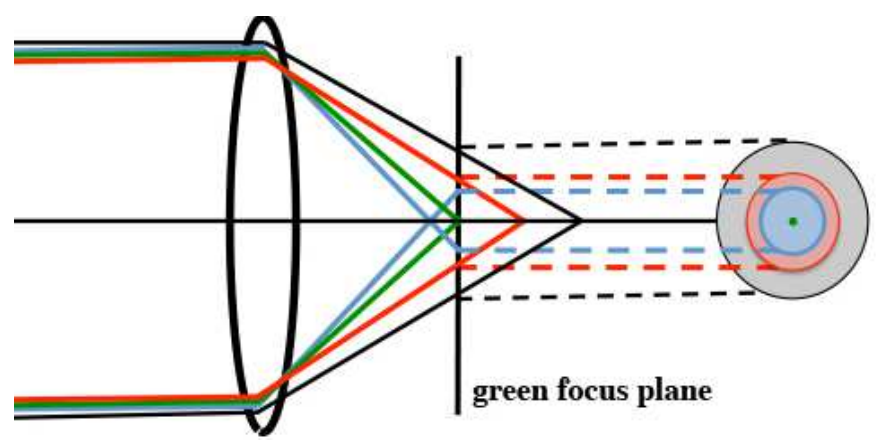

Fig. 1. With a simple lens, every wavelength has its own focal distance, causing different magnitudes of blur for different spectral bands. Taken from [17] with authors' permission.

If one of the color channels is in focus on the sensor plane, the images of all other channels are blurred. This distortion is most noticeable in the NIR image as the wavelength range of the NIR radiation is most different from that of the color channels.

Out-of-focus NIR images can be deblurred through postprocessing [14-16]. In [17], Sadeghipoor et al. suggest that the gradients of the luma channel $Y$ (the pixel-wise average of color channels) and of the NIR image representing the same scene are generally similar. Their algorithm matches the gradient of the NIR image iteratively over multiple scales of resolution to the gradient of the $Y$ image, thus deblurring the NIR image using the high-frequency content in $Y$.

In this paper we present a novel approach that leverages the correlation between each color channel and NIR and takes advantage of the chromatic aberration across channels to deblur the NIR image. Our algorithm corrects for the chromatic aberration in the NIR image and extends its depth-of-field. The proposed method improves upon the state-of-the-art technique presented in [17] by separately exploring all color channels and their spectral and spatial correlations to obtain better deblurring, and by increasing the depth-of-field of the deblurred NIR as well. Section 2 presents a study of correlation among different spectral channels. The proposed algorithm is explained in Section 3. The experimental results showing the improvement of our method over the state of the art are reported in Section 4. 
Table 1. Average sharpness values of different channels.

\begin{tabular}{|c|c|c|c|c|}
\hline & $\mathrm{R}$ & $\mathrm{G}$ & $\mathrm{B}$ & $\mathrm{Y}$ \\
\hline Crete & 0.6235 & 0.5942 & 0.6196 & $\mathbf{0 . 5 1 1 4}$ \\
\hline
\end{tabular}

\section{SPECTRAL-SPATIAL CORRELATION}

In the following subsections, we analyze the correlation between color and NIR channels, their sharpness properties and how these are affected by chromatic aberration distortions.

\subsection{Sharpness and spatial correlation}

To assess the image sharpness we use the measure presented in [18], which we refer to as the Crete sharpness. It is computed based on the idea that blurring a sharp image with a Gaussian kernel causes a more significant loss in normalized gradients compared to blurring an already blurred image.

As extensively discussed and exploited in the literature, the edges of different color channels are usually co-located [17,19-21]. However, the magnitude of gradient varies across different color channels depending on the object reflectance properties. Fig. 2 (c) illustrates an example of the differences in gradient magnitude between red and green channels. In Section 3, we describe how our algorithm exploits these differences in sharpness.

As mentioned before, the algorithm proposed in [17] uses only the $Y$ channel (an average of color channels) to deblur the NIR image. In Table 1, we report the sharpness values of different spectral channels averaged over a dataset of $73 \mathrm{im}-$ ages. This analysis shows that the sharpness values are consistently higher in the color channels than in the $Y$ channel. Motivated by these results, and to leverage the different spectral correlations, we propose using all three color channels in deblurring the NIR image.

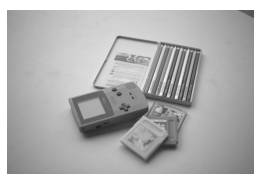

(a) Red channel

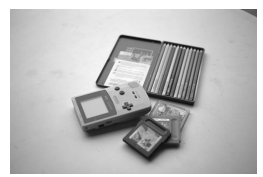

(b) Green channel

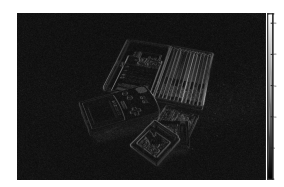

(c) Gradient diff.
Fig. 2. (a) Red channel. (b) Green channel. (c) Absolute difference of vertical Sobel gradients of red and green images (a gamma correction of 0.5 is used for illustration).

\subsection{Spectral correlation}

The high-frequency components are the most distorted as a result of chromatic aberration. They are also the most correlated across spectral bands. We use the normalized crosscorrelation (NCC) to study the correlation between NIR and $Y$ channels. A cross-correlation map (NCC-map) is obtained by computing the NCC score for $20 \times 20$ patches taken from the NIR and $Y$ images. These patches partition the entire image with an overlap of $80 \%$ for a smoother result. A sample result is shown in Fig. 3 (c).

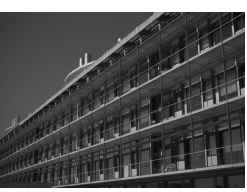

(a) $Y$

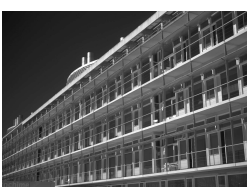

(b) NIR

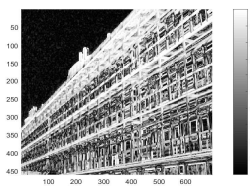

(c) NCC-map
Fig. 3. (a) $Y$ image. (b) NIR with same focus plane as the color image. (c) Absolute NCC-map.

It can be seen in Fig. 3 (c) that the high-frequency regions (edges and fine details in the image) are strongly correlated. In low-frequency regions (uniform patches in the image), normalized cross-correlation effectively looks at the correlation between the noise present in the two spectral channels, which is almost uncorrelated. In contrast, high-frequency components of the image dominate the noise component around the edges resulting in large NCC scores for those regions. The same conclusion can be drawn from the NCC-maps shown in Fig. 4. More importantly, the differences between top and bottom rows of Fig. 4 highlight the fact that the correlation between NIR and different color channels is not identical across a given image.

\subsection{Spectral correlation in the presence of chromatic aberration}

In Table 2, we summarize the normalized cross-correlation between different spectral channels. These results are computed over a dataset of 73 images that suffer from chromatic aberration distortions. As can be seen in this table, the NCC scores are smaller for the channels that are spectrally further apart from each other. This is partly explained by the fact that the refractive index of the lens decreases with the increase in wavelength of the incoming light. Therefore the difference in distance of the focus planes for channels that are spectrally close is smaller. As a result, the objects that are perfectly in focus in the NIR image are likely to still be sharp in the red

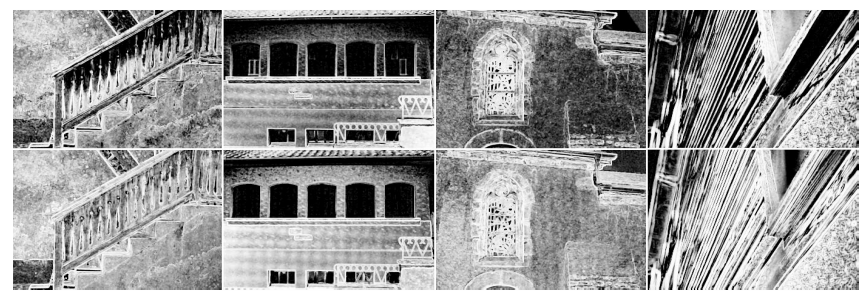

Fig. 4. Top row: NCC maps between NIR and blue channels. Bottom row: NCC maps between NIR and red channels. 
Table 2. NCC values between different spectral channels.

\begin{tabular}{|c|c|c|c|c|}
\hline & NIR & R & G & B \\
\hline NIR & 1 & 0.8436 & 0.7938 & 0.6975 \\
\hline R & - & 1 & 0.9215 & 0.8510 \\
\hline G & - & - & 1 & 0.9310 \\
\hline B & - & - & - & 1 \\
\hline
\end{tabular}

image, while they may be blurred in the green and blue channels, and vice versa. In the next section we describe how our algorithm benefits from this observation.

\section{MULTI-SPECTRAL DEBLURRING ALGORITHM}

From the analysis in Section 2, we conclude that to obtain a good deblurred image using different spectral channels, the most important image components are the high frequencies. Such sharp regions are mostly present in the visible color bands that are in focus. However, for any given region the sharpest details might be found in different spectral channels depending on the reflectance properties of the objects present in the scene. The refractive index of a simple lens decreases from blue wavelength to NIR. The focus plane of the NIR is thus at a larger distance compared to that of the blue channel.

As the sharpest details appear in different spatial locations and in different spectral channels, our proposed algorithm uses all of the available spectral channels for deblurring the NIR image.

The algorithm we propose is based on the deblurring technique introduced in [17], which we refer to as MSDeblur. As shown in (1), MSDeblur takes as its inputs one out-of-focus image $N I R_{b}$ and one guide image $W$ and returns a deblurred image $N I R_{W}$ based on the provided guide $W$. While in [17] only the $Y$ channel is used as a guide, we propose to produce three different deblurred NIR images. We generate the three deblurred images, $N I R_{W}$ for $W \in\{R, G, B\}$, by deblurring $N I R_{b}$ based on the technique described in [17].

$$
N I R_{W}=\operatorname{MSDeblur}\left(N I R_{b}, W\right)
$$

The Crete sharpness measure is then computed over $10 \times 10$ patches across the images $N I R_{b}, N I R_{R}, N I R_{G}$ and $N I R_{B}$. The final deblurred image is reconstructed by selecting the patches with the highest sharpness out of the four intermediate images as shown in (2) where $(p)$ refers to one patch in the image, and Sharp to a sharpness function that returns the sharpness value of its argument (see Fig. 5).

$$
N I R_{\text {final }}^{(p)}=\underset{Z \in R, G, B, b}{\operatorname{argmax}}\left(\operatorname{Sharp}\left(N I R_{Z}^{(p)}\right)\right)
$$

If two or more patches have the same sharpness, the one obtained by the guide image spectrally closest to NIR is chosen as it has the highest a priori probability of correlation to NIR.

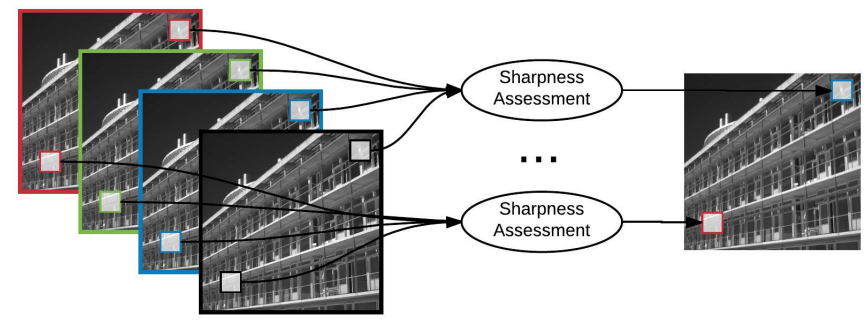

Fig. 5. The images inside red, green and blue boxes are the results of deblurring the NIR image with MSDeblur using the corresponding color images as guides. The image with a black box is the captured NIR image. The Crete sharpness is computed for each patch in these four images and the sharpest patches are used to reconstruct the final NIR image.

With this approach, we exploit the sharpest details across all spectral channels in deblurring the NIR image. Furthermore, spatial regions that are sharper in $N I R_{b}$ than in color channels, due to depth-dependent chromatic aberration, are also considered.

In a typical image, the green channel is focused around mid-range depth, so the blue channel is focused on slightly closer objects, the red channel on objects slightly further away, and the blurred NIR channel is focused on objects that are distant in the scene. Our approach improves on [17] by preserving the details where the NIR image is sharp, and by deblurring the rest of the image using the most appropriate channel depending on the physical (reflectance) and geometric (depth) characteristics of objects present in the scene.

\section{EXPERIMENTAL RESULTS}

The proposed algorithm is tested on a dataset of 73 images taken with a Canon Rebel T1i camera. Without any NIRblocking filter, the camera's silicon sensor detects light waves from approximately $350 \mathrm{~nm}$ to $1000 \mathrm{~nm}$. To capture the color images, an infrared-blocking filter is mounted on the lens. Similarly, a visible-blocking filter is mounted on the lens to capture NIR images. Every scene is captured three times, first the color image is focused at some depth and captured, then the NIR image is captured with the same focus settings, and lastly the camera is refocused to capture a second NIR image that is focused at the same depth the color image was focused on. The second NIR image is only used for comparisons as it cannot be acquired in joint acquisition.

We use the same parameters for MSDeblur as those set in [17] for all three guide channels. The results obtained for the full dataset are assessed in terms of the image sharpness using the Crete sharpness measure and average values are reported in Table 3. Our method improves on the results of [17] by an increase of $48.8 \%$ in terms of the Crete sharpness.

Unlike [17], we make use of the chromatic aberration present between color channels and of the spatial differences 
in high-frequency components by leveraging the sharpest channel at every location (see Fig. 6). We also preserve the high-frequency components of the NIR channel in distant regions, where NIR is the spectral band in focus (see Fig. 7). This allows our algorithm to benefit from the chromatic aberration naturally present in the NIR image to get sharp results for objects further away from the camera.

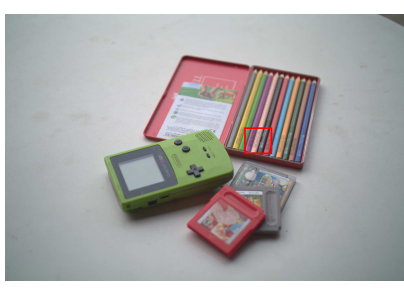

(a) Color image (red box around the cropped region)

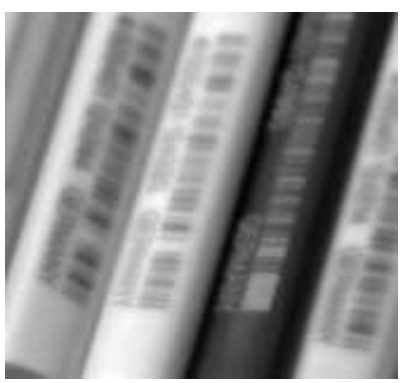

(c) Result from [17]

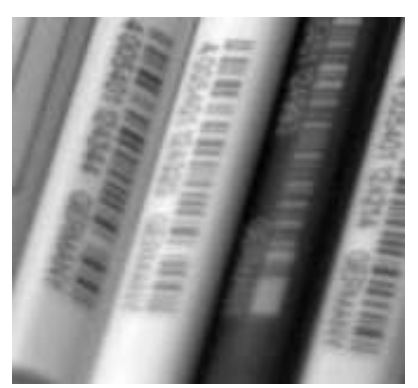

(b) Crop region from $N I R_{b}$

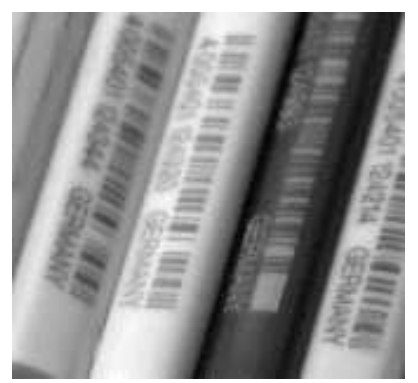

(d) Our result
Fig. 6. (a) Color image. (b) Zoomed-in crop from the corresponding blurred NIR image. (c) Result of deblurring obtained by the algorithm of [17]. (d) Result of our algorithm.

In Fig. 6, we can see the improvement of deblurring with our proposed method, compared to the results obtained in [17]. The pencils in the image reflect different colors and therefore the high-frequency components in that region of the image are non-uniformly distributed over the different color bands. Fig. 7 shows how our approach can also extend the depth-of-field of the NIR images. The NIR image captured with focus settings used for the color image is out of focus on the plane where the color image is focused. However, due to the smaller refractive index, the NIR image is in focus on the distant background. By preserving this sharp region, we obtain a better final result that is in focus on the focus planes of all spectral channels, color and NIR. As a result, the deblurred NIR image has a larger depth-of-field. Additional results are shown in Fig. 8 comparing our results to those of [17]. The first image (left to right) is sharper everywhere. In the second image the improvement is most visible around the lantern and its hanging bar. And in the last two images it is the text that becomes more readable and the gray bars that become sharper.

We obtain similar results using Sobel gradients as a sharp-

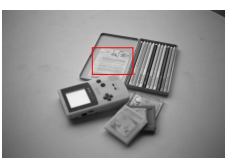

(a) $N I R_{b}$ with crop box

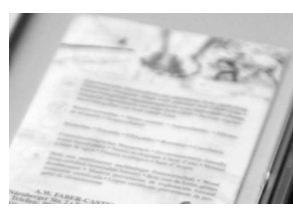

(b) In-focus NIR

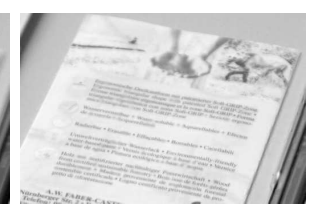

(c) Our result
Fig. 7. (a) $N I R_{b}$ blurred image. (b) NIR image taken after refocusing the camera for NIR capture. (c) $N I R_{b}$ deblurred with our approach, with extended depth-of-field compared to the in-focus NIR image.

Table 3. Crete sharpness averaged over the dataset.

\begin{tabular}{|l|c|c|}
\hline & Result of [17] & Our result \\
\hline Crete sharpness & 0.3253 & $\mathbf{0 . 4 8 4 1}$ \\
\hline
\end{tabular}

ness measure. Sobel sharpness is computed over a specified region as the absolute sum of gradients obtained through 2Dconvolution with Sobel kernels. Recombining the images in (2) based on Sobel sharpness then studying the Crete sharpness of the output, or vice versa, as well as doing both steps with Sobel sharpness, all yield closely similar performance and improvement.

\section{CONCLUSION}

We studied the cross-correlation between spectral channels in the joint acquisition of color and NIR images. Highfrequency components having the strongest inter-spectral cross-correlation are found in different spatial-spectral coordinates, as a result of depth-varying chromatic aberration and reflectance properties of scene objects.

We proposed a novel technique that leverages these different correlation types as well as chromatic aberration to deblur out-of-focus NIR images. Future work can use the technique proposed in this paper to take advantage of the in-focus distant regions in the NIR image for deblurring color images. Due to chromatic aberration and the smaller refractive index of NIR, these regions can be sharp in the NIR image and thus a valuable reference for deblurring the color counterparts.

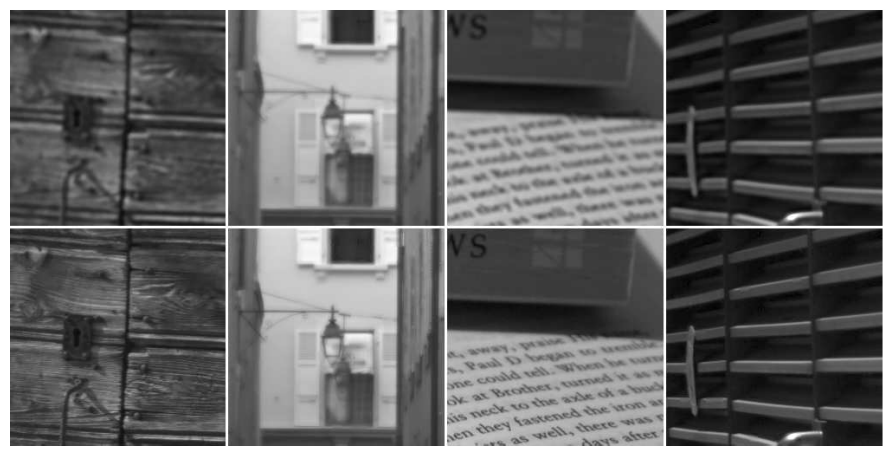

Fig. 8. Comparison between results obtained by the algorithm of [17] (top row) and our algorithm (bottom row). 


\section{REFERENCES}

[1] C. Fredembach and S. Süsstrunk, "Colouring the nearinfrared," in Color and Imaging Conference. Society for Imaging Science and Technology, 2008, pp. 176-182.

[2] D. Rüfenacht, C. Fredembach, and S. Süsstrunk, "Automatic and accurate shadow detection using near-infrared information," IEEE Transactions on Pattern Analysis and Machine Intelligence, vol. 36, no. 8, pp. 1672-1678, 2014.

[3] M. Brown and S. Süsstrunk, "Multi-spectral sift for scene category recognition," in Proc. IEEE Conference on Computer Vision and Pattern Recognition (CVPR), 2011, pp. 177-184.

[4] S. Z Li, R. Chu, S. Liao, and L. Zhang, "Illumination invariant face recognition using near-infrared images," IEEE Transactions on Pattern Analysis and Machine Intelligence, vol. 29, no. 4, pp. 627-639, 2007.

[5] G. K. Naganathan, L. M Grimes, J. Subbiah, C. R Calkins, A. Samal, and G. E Meyer, "Visible/nearinfrared hyperspectral imaging for beef tenderness prediction," Computers and Electronics in Agriculture, vol. 64, no. 2, pp. 225-233, 2008.

[6] R. Rossel, D. Walvoort, A. McBratney, L. J Janik, and J. O Skjemstad, "Visible, near infrared, mid infrared or combined diffuse reflectance spectroscopy for simultaneous assessment of various soil properties," Geoderma, vol. 131, no. 1, pp. 59-75, 2006.

[7] M. Shibayama and T. Akiyama, "Seasonal visible, nearinfrared and mid-infrared spectra of rice canopies in relation to lai and above-ground dry phytomass," Remote Sensing of Environment, vol. 27, no. 2, pp. 119-127, 1989.

[8] D. Krishnan and R. Fergus, "Dark flash photography," in ACM Transactions on Graphics, 2009, vol. 28, p. 96.

[9] L. Schaul, C. Fredembach, and S. Süsstrunk, "Color image dehazing using the near-infrared," in Proc. IEEE International Conference on Image Processing (ICIP), 2009, pp. 1629-1632.

[10] Y. M Lu, C. Fredembach, M. Vetterli, and S. Süsstrunk, "Designing color filter arrays for the joint capture of visible and near-infrared images," in Proc. IEEE International Conference on Image Processing (ICIP), 2009, pp. 3797-3800.

[11] G. Langfelder, T. Malzbender, A. Longoni, and F. Zaraga, "A device and an algorithm for the separation of visible and near infrared signals in a monolithic silicon sensor," in IS\&T/SPIE Electronic Imaging, 2011, pp. 788207-788207.
[12] Z. Sadeghipoor, Y. M Lu, and S. Süsstrunk, "A novel compressive sensing approach to simultaneously acquire color and near-infrared images on a single sensor," in Proc. IEEE International Conference on Acoustics, Speech and Signal Processing (ICASSP), 2013, pp. 1646-1650.

[13] X. Luo, J. Zhang, and Q. Dai, "Hybrid fusion and demosaicing algorithm with near-infrared image," in SPIE Sensing Technology+ Applications, 2014, pp. 91210J91210J.

[14] Z. Sadeghipoor, Y. M Lu, and S. Süsstrunk, “Gradientbased correction of chromatic aberration in the joint acquisition of color and near-infrared images," in SPIE/IS\&T Electronic Imaging, 2015, pp. 94040F94040F.

[15] D. Krishnan, T. Tay, and R. Fergus, "Blind deconvolution using a normalized sparsity measure," in Proc. IEEE Conference on Computer Vision and Pattern Recognition (CVPR), 2011, pp. 233-240.

[16] K. He, J. Sun, and X. Tang, "Guided image filtering," IEEE Transactions on Pattern Analysis and Machine Intelligence, vol. 35, no. 6, pp. 1397-1409, 2013.

[17] Z. Sadeghipoor, Y. M Lu, E. Mendez, and S. Süsstrunk, "Multiscale guided deblurring: Chromatic aberration correction in color and near-infrared imaging," in 23rd European Signal Processing Conference (EUSIPCO), 2015, pp. 2336-2340.

[18] F. Crete, T. Dolmiere, P. Ladret, and M. Nicolas, "The blur effect: perception and estimation with a new noreference perceptual blur metric," in Electronic Imaging, 2007, pp. 64920I-64920I.

[19] S. B. Kang, "Automatic removal of chromatic aberration from a single image," in Proc. IEEE Conference on Computer Vision and Pattern Recognition (CVPR), 2007, pp. 1-8.

[20] S. Chung, B. Kim, and W. Song, "Detecting and eliminating chromatic aberration in digital images," in Proc. IEEE International Conference on Image Processing (ICIP), 2009, pp. 3905-3908.

[21] F. Heide, M. Rouf, M. B Hullin, B. Labitzke, W. Heidrich, and A. Kolb, "High-quality computational imaging through simple lenses," ACM Transactions on Graphics (TOG), vol. 32, no. 5, pp. 149, 2013. 